\section{Journal of \\ Epilepsy and \\ Clinical \\ Neurophysiology}

J Epilepsy Clin Neurophysiol 2009;15(3):123-129

\title{
Adaptação Transcultural do Inventário Neurocomportamental (NBI) para o Brasil
}

\author{
Guilherme Nogueira Mendes de Oliveira*, Arthur Kummer**, João Vinícius Salgado***, \\ Eduardo Jardel Portela****, Sílvio Roberto Sousa-Pereira****, Mirian Fabíola Studart Gurgel Mendes****, \\ Renato Luiz Marchetti*****, Antônio Lúcio Teixeira******
}

Núcleo de Neuropsiquiatria do Serviço de Neurologia do Hospital das Clínicas da Universidade Federal de Minas Gerais (UFMG)

\section{RESUMO}

Objetivo: Realizar a adaptação transcultural de instrumento para avaliação das alterações comportamentais tipicamente descritas em portadores de epilepsia do lobo temporal (ELT), o Inventário Neuro-Comportamental (NBI) para a população brasileira. Métodos: Inicialmente, foi feita a tradução do instrumento original para o português. Esta versão foi revisada e retrotraduzida para o inglês. A seguir, a versão retrotraduzida foi comparada à versão original em inglês, sendo corrigidas as divergências no texto em português. Em um segundo momento, 15 pacientes do Ambulatório de Epilepsia do Hospital das Clínicas da UFMG portadores de ELT responderam ao inventário. À aplicação do questionário, eventuais dificuldades e os itens mal compreendidos foram analisados pelos autores. Resultados: Na versão final para o português, os itens 11, 14, 17, 61 e 75 foram modificados. Sete pacientes (46,7\%) eram do sexo feminino, com idade entre 26 e 65 anos. A maioria dos pacientes $(93,3 \%)$ apresentou uma pontuação total elevada. Os domínios mais comumente alterados foram hiperreligiosidade, detalhismo e crença na predestinação pessoal (73,3\% dos pacientes em cada um deles). Conclusão: A versão em português do NBI pode ser um instrumento útil para avaliar alterações comportamentais na ELT aplicada ao contexto clínico dos pacientes brasileiros.

Unitermos: epilepsia, epilepsia do lobo temporal, comportamento, NBI, adaptação transcultural.

\section{ABSTRACT}

Cross-cultural adaptation of the Neurobehavior Inventory (NBI) for Brazil

Purpose: To perform the cross-cultural adaptation of an instrument designed to measure behavioral changes typically described in temporal lobe epilepsy (TLE), the Neurobehavior Inventory (NBI), for Brazilian population. Methods: At first the original instrument was translated to Portuguese-Brazilian language. This version was revised and translated back into English. Later, both English versions were compared and the divergences were corrected in the Portuguese text. The second step consisted of the application of the inventory to 15 TLE patients from the Epilepsy Program, Federal University of Minas Gerais. The authors analyzed eventual difficulties and misunderstandings in the application of the questionnaire. Results: In the final Portuguese version, the questions $11,14,17,61$ and 75 were considered difficult to understand and were modified. Seven (46.7\%) subjects were women, with 26 to 65 years. Most of the patients $(93.3 \%)$ had a high total score level.

* Médico Psiquiatra. Pós-Graduando em Neurociências pela Universidade Federal de Minas Gerais (UFMG). Colaborador do Grupo de Epilepsia do Serviço de Neurologia do Hospital das Clínicas da UFMG, Belo Horizonte. Professor de Saúde Mental do Centro Universitário de Belo Horizonte - Uni-BH.

** Médico Psiquiatra. Doutor em Neurociências pela Universidade Federal de Minas Gerais (UFMG). Colaborador do Grupo de Epilepsia do Serviço de Neurologia do Hospital das Clínicas da UFMG, Belo Horizonte.

*** Médico Psiquiatra. Doutor em Neurociências pela Universidade Louis Pasteur, Estrasburgo, França e pela Universidade de São Paulo, Ribeirão Preto. Professor do Instituto de Ciências Biológicas (ICB) da Universidade Federal de Minas Gerais (UFMG), Belo Horizonte.

***** Médico Neurologista. Grupo de Epilepsia do Serviço de Neurologia do Hospital das Clínicas da Universidade Federal de Minas Gerais (UFMG).

***** Médico Psiquiatra. Doutor em Psiquiatria pela Universidade de São Paulo. Coordenador do Projeto de Epilepsia e Psiquiatria (PROJEPSI) do Instituto de Psiquiatria do Hospital das Clínicas da Faculdade de Medicina da Universidade de São Paulo (IPq-HCFMUSP).

****** Médico Neurologista e Psiquiatra. Doutor em Biologia Celular. Professor da Faculdade de Medicina da Universidade Federal de Minas Gerais (UFMG), Belo Horizonte.

Received June 16, 2009; accepted July 24, 2009. 
Religious convictions, interest in details and sense of personal destiny were the most common altered domains (73.3\% of the patients in each one of them). Conclusion: The Portuguese-Brazilian version of the NBI may be a useful instrument to evaluate TLE behavioral alterations in the Brazilian clinical context.

Key words: epilepsy, temporal lobe epilepsy, behavior, NBI, cross-cultural adaptation.

\section{INTRODUÇÃO}

A epilepsia do lobo temporal (ELT) é a forma de epilepsia mais comum no adulto, correspondendo a aproximadamente $40 \%$ de todos os casos de epilepsia. Pacientes com ELT apresentam maiores taxas de comorbidades psiquiátricas como depressão e psicose, quando comparados a pacientes com outros tipos de epilepsia. ${ }^{7}$ Apesar de bastante frequentes, os transtornos psiquiátricos são ainda subdiagnosticados e subtratados na maioria dos pacientes com epilepsia. ${ }^{7,9}$

Há mais de 3.000 anos, escritos babilônicos já correlacionavam sintomas psicóticos e alterações comportamentais, como hipossexualidade e hiperreligiosidade, à epilepsia. ${ }^{11}$ No entanto, ainda hoje, os debates acerca do comportamento interictal em portadores de ELT são permeados por muita controvérsia. ${ }^{12}$

Em meados do século XX, Gastaut observou que a intensidade emocional, "viscosidade" (ações e fala com ordenação e detalhes excessivos) e hipossexualidade (diminuição no interesse e desejo sexuais), comuns em pacientes com ELT, eram exatamente opostas à placidez, dispersão atencional e hipersexualidade apresentadas por pacientes submetidos à lobectomia temporal bilateral, quadro denominado de síndrome de Klüver-Bucy. ${ }^{3,4}$ Posteriormente, as contribuições de Geschwind foram importantes para descrever outras características frequentes em pacientes com ELT, como o temperamento lábil, a tendência de escrever extensivamente (hipergrafia) e preocupações exacerbadas com questões éticas e religiosas. Assim, esse quadro comportamental interictal passou a ser conhecido por síndrome de Gastaut-Geschwind. ${ }^{10,12}$

É importante relatar o esforço de pesquisadores na elaboração de ferramentas clínicas capazes de identificarem e quantificarem as características comportamentais mais comuns na ELT. Inicialmente foi desenvolvido o Inventário de Bear e Fedio (IBF) para avaliar 18 traços (cada um composto por 5 itens) de personalidade associados à ELT, além de 10 itens modificados do Minnesota Multiphasic Personality Inventory (MMPI). ${ }^{12}$ Blummer et al. (1995), após experiências com o IBF, propuseram alterações neste instrumento. Retiraram os 10 itens do MMPI, acrescentaram 5 novas afirmativas sobre bem-estar físico relacionadas a sintomas somáticos depressivos e 5 novas afirmativas sobre medo e desconfiança; revisaram ainda todos os itens sobre sexualidade a fim de explorar especificamente hipossexualidade, desenvolvendo assim o Inventário Neuro-Comportamental (NBI). ${ }^{3}$

Outros questionários foram propostos, como a versão da Entrevista Clínica Estruturada do Diagnostic and Statistical Manual of Mental Disorders (DSM) para a epilepsia (SCID-E), mas ainda não existe um único instrumento que avalie de forma ampla, confiável e reprodutível as alterações comportamentais nessa população. Nesse contexto e considerando a dificuldade intrínseca em se objetivar um assunto tão complexo como o comportamento, o NBI pode ser considerado o teste que mais se aproxima de um padrão-ouro. $^{8}$

Portanto, o objetivo deste trabalho é contribuir para o estudo neuropsiquiátrico das epilepsias no contexto brasileiro através da adaptação à nossa realidade do instrumento clínico que provavelmente investiga de maneira mais completa as alterações comportamentais encontradas nesses pacientes, o NBI.

\section{MÉTODOS}

\section{Instrumento}

O NBI avalia características da personalidade (viscosidade, emoções, consciência, espiritualidade, hipossexualidade e dependência), assim como estados episódicos que podem ter relevância clínica (humor, irritabilidade, paranóia e ansiedade). O inventário é composto por 100 itens que devem ser assinalados como verdadeiros ou falsos. Cada grupo de 5 afirmativas investiga uma dos seguintes domínios (os números entre parênteses correspondem a cada uma das afirmativas incluídas no $\mathrm{NBI})$ : tendência à escrita $(6,18,53,62,98)$, hipermoralismo $(19,41,49,75,83)$, convicções religiosas $(13,24,48,94,99)$, raiva e temperamento $(20,25,36,43,91)$, tendência à sistematização ou à ordenação $(5,14,21,42,71)$, hipossexualidade $(7,10,52,77,84)$, medo e insegurança $(16,40,57,64,86)$, sentimentos de culpa $(4,55,61,70,80)$, seriedade $(29,45,66,88,100)$, tristeza $(33,38,73,85,92)$, emotividade $(9,23,54,69,89)$, desconfiança e suspeição $(12,17,22,34,63)$, detalhismo $(26,50,58,67,81)$, interesses cósmicos $(37,46,51,72,76)$, crença na predestinação pessoal $(1,11,27,32,97)$, persistência e repetitividade $(35,44,60,78,90)$, ódio e vingança $(56,68,82,87,96)$, dependência $(3,15,28,39,93)$, euforia $(2,31,59,65,79)$ e 
somatização $(8,30,47,74,95)$. Mais de dois itens marcados como verdadeiros em cada um dos domínios ou uma soma total superior a vinte itens verdadeiros são valores considerados elevados. ${ }^{3,8}$

\section{Tradução, retrotradução e versão final}

Primeiramente, Dr. Renato Luiz Marchetti realizou contato com o autor do NBI, Prof. Dietrich Blumer, que autorizou a tradução do inventário para o português.

Para produzir o NBI em português, um dos autores fez a tradução inicial que foi revisada. A versão revisada foi retrotraduzida para o inglês por outro autor. A seguir, a versão retrotraduzida foi comparada à versão original em inglês. Ao compará-las, foram corrigidas eventuais divergências no texto em português para que fosse mantido o significado proposto pelo autor do inventário, chegandose à versão final em português utilizada na fase de teste. ${ }^{3}$

\section{Fase de teste}

Foram selecionados quinze pacientes portadores de ELT para a fase de teste, em tratamento regular no ambulatório de Epilepsia do Hospital das Clínicas da UFMG, que deram seu livre consentimento para participar do estudo. Foram excluídos os portadores de transtornos mentais do eixo I do DSM à entrevista psiquiátrica padronizada pelo M.I.N.I. Plus 5.0.0. ${ }^{1}$ Também foram excluídos os portadores de demência ou declínio cognitivo conforme pontuação no Miniexame do Estado Mental (MEEM). ${ }^{5}$

À medida que os pacientes respondiam ao questionário comentavam sobre eventuais dificuldades no seu entendimento e os itens mal compreendidos foram ana- lisados pelos autores. O objetivo dessa etapa foi verificar se as questões estavam claramente expressas e condizentes com nossa cultura.

\section{RESULTADOS}

Em reunião entre os autores, foram discutidos e modificados os itens: 11, 14, 17, 61 e 75 (Tabela 1), chegando-se à versão final em português apresentada no final deste artigo (Anexo 1). Em relação às modificações, na questão 11, foi substituída a palavra SUPREMO, por não ser de uso frequente e de difícil compreensão por alguns entrevistados. As demais questões sofreram adaptações mais livres já que sua tradução literal não expressava sua real intenção e comprometia o sentido pretendido.

As características clínicas dos 15 pacientes estudados são mostradas na Tabela 2. Sete pacientes (46,7\%) eram do sexo feminino, com idade entre 26 e 65 anos. Oito (53.4\%) haviam cursado até o ensino fundamental (Tabela 2).

A grande maioria dos pacientes apresentou uma pontuação total elevada, sendo que catorze $(93,3 \%)$ dos quinze pacientes apresentaram escore final acima de 20 pontos: mediana (min-máx) = 44 (17-82); média $(D P)=45,13(16,62)$. Como mostrado na Tabela 3 , hiperreligiosidade, detalhismo e crença na predestinação pessoal foram os domínios mais comumente encontrados $(73,3 \%)$, mas também hipermoralismo $(66,7 \%)$, seriedade e interesses cósmicos (60\%). Por outro lado, foi rara a ocorrência de tristeza e sentimento de ódio e vingança $(13,3 \%)$, o que possivelmente reflete a exclusão daqueles portadores de transtornos mentais do eixo I do DSM, como depressão maior (Tabela 3).

Tabela 1. Modificações dos autores após propostas dos pacientes.

\begin{tabular}{cll}
\hline Questão & \multicolumn{1}{c}{ Versão original } & \multicolumn{1}{c}{ Versão final em português } \\
\hline 11 & I believe that I serve a SUPREME purpose in life. & Eu acredito que sirvo a um propósito ESPECIAL na vida. \\
14 & I am more sensitive to distractions than most people. & Eu me distraio mais facilmente do que a maioria das pessoas. \\
17 & I am open to attack from many sides. & Eu desconfio que possa ser atacado (a) de muitos lados \\
61 & I have not lived the right kind of life. & Eu não tenho vivido a vida da maneira correta. \\
75 & I would go out of my way to make sure the law is followed. & Eu faria o que for preciso para ter certeza de que a lei está sendo cumprida. \\
\hline
\end{tabular}

Tabela 2. Características demográficas dos pacientes avaliados.

\begin{tabular}{lc}
\hline \multicolumn{1}{c}{ Características } & Pacientes (15) \\
\hline Sexo & $8 \mathrm{M} / 7 \mathrm{~F}$ \\
Idade (anos) - média (DP) & $44,53(10,26)$ \\
Escolaridade (anos) - média (DP) & $7,40(3,71)$ \\
MEEM - média (DP) & $27,47(2,61)$ \\
Calendário das crises (crises/mês) - média (DP) & $5,04(5,74)$ \\
Tempo de diagnóstico (anos) - média (DP) & $35,00(12,53)$ \\
\hline
\end{tabular}

M - masculino; F - feminino; DP - desvio-padrão; MEEM - miniexame do estado mental. 
Tabela 3. Pontuação nos domínios comportamentais avaliados pelo NBI.

\begin{tabular}{|c|c|c|c|}
\hline Domínio (itens) & Mediana (min-máx) & Média (DP) & $\begin{array}{c}\text { >2itens/domínio } \\
(\%)\end{array}$ \\
\hline Tendência à escrita $(6,18,53,62,98)$ & $1,00(0-5)$ & $1,87(1,45)$ & $33,3 \%$ \\
\hline Hipermoralismo $(19,41,49,75,83)$ & $3,00(1-5)$ & $2,93(1,22)$ & $66,7 \%$ \\
\hline Convicções religiosas $(13,24,48,94,99)$ & $4,00(0-5)$ & $3,53(1,45)$ & $73,3 \%$ \\
\hline Raiva e temperamento $(20,25,36,43,91)$ & $1,00(0-4)$ & $1,27(1,33)$ & $20 \%$ \\
\hline Tendência à sistematização ou à ordenação $(5,14,21,42,71)$ & $3,00(0-4)$ & $2,33(1,44)$ & $53,3 \%$ \\
\hline Hipossexualidade $(7,10,52,77,84)$ & $2,00(0-5)$ & $2,40(1,63)$ & $40 \%$ \\
\hline Medo e insegurança $(16,40,57,64,86)$ & $2,00(1-4)$ & $2,33(, 90)$ & $46,7 \%$ \\
\hline Sentimentos de culpa $(4,55,61,70,80)$ & $2,00(0-4)$ & $2,13(1,30)$ & $46,7 \%$ \\
\hline Seriedade $(29,45,66,88,100)$ & $4,00(2-5)$ & $3,33(1,23)$ & $60 \%$ \\
\hline Tristeza $(33,38,73,85,92)$ & ,00 (0-4) & $1,00(1,30)$ & $13,3 \%$ \\
\hline Emotividade $(9,23,54,69,89)$ & $1,00(0-5)$ & $2,00(1,41)$ & $40 \%$ \\
\hline Desconfiança e suspeição $(12,17,22,34,63)$ & $1,00(0-4)$ & $1,33(1,49)$ & $20 \%$ \\
\hline Detalhismo $(26,50,58,67,81)$ & $3,00(1-5)$ & $3,20(1,20)$ & $73,3 \%$ \\
\hline Interesses cósmicos $(37,46,51,72,76)$ & $3,00(0-5)$ & $2,67(1,54)$ & $60 \%$ \\
\hline Crença na predestinação pessoal $(1,11,27,32,97)$ & $3,00(0-5)$ & $3,13(1,40)$ & $73,3 \%$ \\
\hline Persistência e repetitividade $(35,44,60,78,90)$ & $3,00(0-5)$ & $2,47(1,92)$ & $53,3 \%$ \\
\hline Ódio e vingança $(56,68,82,87,96)$ & $1,00(0-3)$ & $1,20(1,08)$ & $13,3 \%$ \\
\hline Dependência $(3,15,28,39,93)$ & $2,00(0-4)$ & $1,93(1,16)$ & $26,7 \%$ \\
\hline Euforia $(2,31,59,65,79)$ & $2,00(1-5)$ & $2,20(1,08)$ & $33,3 \%$ \\
\hline Somatização $(8,30,47,74,95)$ & $2,00(0-4)$ & $1,87(1,06)$ & $26,7 \%$ \\
\hline
\end{tabular}

\section{DISCUSSÃO}

De maneira geral a versão brasileira do NBI foi bem compreendida e aceita pela maioria dos pacientes. No entanto, fica claro que este instrumento é dependente da colaboração e preservação cognitiva dos pacientes, o que pode ser complexo em se tratando de casos refratários e crônicos. O questionário pode ser aplicado também ao acompanhante do paciente e, neste caso, as perguntas são modificadas de modo a avaliar a impressão do acompanhante em relação ao comportamento do paciente. Ambos devem ser entrevistados em casos de divergências para esclarecimento das mesmas. ${ }^{3}$

Os achados demonstram que alterações comportamentais podem ser observadas mesmo nos portadores de ELT em que foram excluídos os transtornos mentais como ansiedade, depressão e psicose. Apesar de uma elevada pontuação total, apenas quatro pacientes apresentaram mais de 9 domínios comportamentais e, destes, somente dois apresentaram 19 e nenhum completou todos os 20 domínios. Apesar de preliminares, esses dados sugerem a obsolescência do termo "personalidade epiléptica", corroborando a opinião de autores que consideram que tais características são incomuns e se manifestam principalmente nos casos com comprometimento significativo de estruturas têmporo-límbicas, refratários e de longa evolução. $3,4,6$ Sob essa perspectiva, é importante ressaltar que todos pacientes incluídos no presente estudo possuíam imagem de ressonância magnética do encéfalo mostrando esclerose hipocampal. Além disso, esses pacientes constituem uma amostra de um centro terciário voltado para o manejo de quadros graves, o que pode ser demonstrado pelo tempo de evolução da doença, média de 35 anos, e pela refratariedade, média de 5 crises ao mês. Os quatro pacientes que obtiveram pontuação elevada em mais de 9 domínios apresentavam um tempo médio de evolução da doença de 41,8 anos.

Finalmente, é relevante contextualizar a pertinência do presente estudo no que tange à recente proposta elaborada pela Comissão de Neuropsiquiatria da ILAE. 2,9 Segundo essa proposta, os atuais sistemas de classificação dos transtornos mentais (DSM-IV e CID-10) não contemplam as particularidades da epilepsia, sendo necessária a elaboração de estratégias e iniciativas específicas que atentem às peculiaridades psicopatológicas dessa população.

\section{CONCLUSÃO}

Embora ainda sejam necessários estudos de validade da versão brasileira do NBI, como o inventário apresenta itens 
bastante genéricos, provavelmente aplicáveis à maioria dos indivíduos, tem o potencial de ser utilizada de modo satisfatório em nosso contexto clínico.

\section{REFERÊNCIAS}

1. Amorim P. Mini International Neuropsychiatric Interview (MINI): validação de entrevista breve para diagnóstico de transtornos mentais. Rev Bras Psiquiatr 2000;22(3):106-15.

2. Araújo Filho GM, Rosa VP, Yacubian EMT. Transtornos psiquiátricos na epilepsia: uma proposta de classificação elaborada pela comissão de neuropsiquiatria da ILAE. J Epilepsy Clin Neurophysiol 2008; 14(3):119-23.

3. Blumer D. Personality disorders in epilepsy. In: Ratey JJ, ed. Neuropsychiatry of personality disorders. Boston: Blackwell Science 1995;230-63.

4. Blumer D. Evidence supporting the temporal lobe epilepsy personality syndrome. Neurology 1999;53(5 Suppl 2):S9-12.

5. Brucki SM, Nitrini R, Caramelli P, Bertolucci PH, Okamoto IH. Suggestions for utilization of the mini-mental state examination in Brazil. Arq Neuropsiquiatr 2003;61(3B):777-81.
6. Devinsky O, Najjar S. Evidence against the existence of a temporal lobe epilepsy personality syndrome. Neurology 1999;53(5 Suppl 2):S13-25.

7. Gaitatzis A, Trimble MR, Sander JW. The psychiatric comorbidity of epilepsy. Acta Neurol Scand 2004;110(4):207-20.

8. Krishnamoorthy ES. The evaluation of behavioral disturbances in epilepsy. Epilepsia 2006;47 (Suppl 2):3-8.

9. Marchetti RL, Castro APW, Kurcgant D, Cremonese E, Gallucci Neto J. Transtornos mentais associados à epilepsia. Rev Psiquiatr Clin 2005;32(3):170-82.

10. Oliveira GNM, Kummer AM, Salgado JV, Marchetti RL, Teixeira AL. Transtornos Neuropsiquiátricos da Epilepsia do Lobo Temporal. Rev Bras Neurol 2009;45(1):15-23.

11. Reynolds EH, Kinnier Wilson JV. Psychoses of epilepsy in Babylon: The oldest account of the disorder. Epilepsia 2008;49(9):1488-90.

12. Shetty T, Trimble M. The Bear Fedio Inventory: Twenty Years On. J Epilepsy 1997;10(5):254-62.

Endereço para correspondência:

Antonio Lucio Teixeira

Departamento de Clínica Médica, Faculdade de Medicina, UFMG

Av. Alfredo Balena, 190 - Santa Efigênia

CEP 30130-100, Belo Horizonte, MG, Brasil

E-mail: altexr@gmail.com

\section{ANEXO 1 \\ INVENTÁRIO NEURO-COMPORTAMENTAL (NBI)}

Nas páginas seguintes serão apresentadas afirmativas sobre atitudes e opiniões pessoais. Para cada afirmativa, por favor, indique se a afirmativa lhe parece verdadeira ou falsa, de acordo com seu ponto de vista.

Assinale no espaço em branco de cada afirmativa, na coluna apropriada de "verdadeiro" $(V)$ ou "falso" $(F)$, sem deixar respostas em branco. Não há respostas certas ou erradas no inventário; o mais importante é a honestidade de suas respostas.

\begin{tabular}{|c|c|c|}
\hline Afirmativas & $\mathbf{V}$ & $\mathbf{F}$ \\
\hline 1. Eu penso que as pessoas aprenderiam muito com a história da minha vida. & & \\
\hline 2. Às vezes tenho sensações intensas de alegria. & & \\
\hline 3. Eu me sinto como um fantoche nas mãos dos outros. & & \\
\hline 4. Eu nunca consigo me perdoar por algumas das coisas que fiz. & & \\
\hline 5. Eu tenho hábito de contar coisas ou memorizar números. & & \\
\hline 6. Faz muito sentido manter um diário detalhado. & & \\
\hline 7. Sexo é menos importante do que a maioria das pessoas acredita. & & \\
\hline 8. Frequentemente eu tenho dificuldades em ter uma boa noite de sono. & & \\
\hline 9. Para mim, sentimentos podem, de repente, tomar o lugar do pensamento. & & \\
\hline 10. Quase nunca eu me preocupo com pensamentos sobre sexo. & & \\
\hline 11. Eu acredito que sirvo a um propósito especial na vida. & & \\
\hline 12. O destino parece agir contra mim. & & \\
\hline 13. Minhas crenças religiosas tornaram-se muito importantes. & & \\
\hline 14. Eu me distraio mais facilmente do que a maioria das pessoas. & & \\
\hline 15. Eu deixei pessoas com raiva por pedi-las que fizessem muito por mim. & & \\
\hline 16. Eu estou muito preocupado (a) em ferir os sentimentos das outras pessoas. & & \\
\hline 17. Eu desconfio que possa ser atacado (a) de muitos lados. & & \\
\hline 18. Eu escrevo poesia, histórias ou biografia. & & \\
\hline 19. Sinto-me pessoalmente furioso (a) ao ver pessoas desobedecendo a lei. & & \\
\hline
\end{tabular}




\begin{tabular}{|c|c|c|}
\hline Afirmativas & $\mathbf{V}$ & $\mathbf{F}$ \\
\hline \multicolumn{3}{|l|}{ 20. Pequenas coisas me fazem mais raiva do que costumavam fazer. } \\
\hline \multicolumn{3}{|l|}{ 21. Se as coisas não estão absolutamente corretas, isso me incomoda. } \\
\hline \multicolumn{3}{|l|}{ 22. As pessoas tendem a tirar vantagem de mim. } \\
\hline \multicolumn{3}{|l|}{ 23. Quase tudo desencadeia alguma reação emocional em mim. } \\
\hline \multicolumn{3}{|l|}{ 24. A Bíblia tem um significado especial que estou começando a entender. } \\
\hline \multicolumn{3}{|l|}{ 25. Meu temperamento tem me causado problemas. } \\
\hline \multicolumn{3}{|l|}{ 26. Algumas vezes eu fico terrivelmente confuso (a) por causa de pequenos detalhes. } \\
\hline \multicolumn{3}{|l|}{ 27. Forças poderosas estão atuando através de mim. } \\
\hline \multicolumn{3}{|l|}{ 28. Eu pareço depender de outras pessoas para muitas coisas. } \\
\hline \multicolumn{3}{|l|}{ 29. Poucas coisas são realmente divertidas. } \\
\hline \multicolumn{3}{|l|}{ 30. Eu estou frequentemente incomodado (a) por graves dores de cabeça ou outras dores. } \\
\hline \multicolumn{3}{|l|}{ 31. Com frequência eu fico tão bem humorado (a) que faço coisas bobas. } \\
\hline \multicolumn{3}{|l|}{ 32. Eu tenho certeza que há um significado importante por trás de meu sofrimento. } \\
\hline \multicolumn{3}{|l|}{ 33. Eu tenho tido períodos de dias ou semanas em que não consigo agir de jeito nenhum. } \\
\hline \multicolumn{3}{|l|}{ 34. Algumas vezes eu escuto sons ou vejo coisas que não estão realmente lá. } \\
\hline \multicolumn{3}{|l|}{ 35. Algumas vezes eu não consigo deixar de lado uma questão. } \\
\hline \multicolumn{3}{|l|}{ 36. Eu estou perdendo o controle do meu temperamento mais frequentemente. } \\
\hline \multicolumn{3}{|l|}{ 37. Nada é mais importante do que tentar entender as forças que governam este mundo. } \\
\hline \multicolumn{3}{|l|}{ 38. A vida é uma tensão para mim na maior parte do tempo. } \\
\hline \multicolumn{3}{|l|}{ 39. Algumas vezes eu me sinto tão desamparado (a) que quero que as pessoas façam tudo para mim. } \\
\hline \multicolumn{3}{|l|}{ 40. Eu posso ficar com medo de estar sozinho (a). } \\
\hline \multicolumn{3}{|l|}{ 41. Com frequência eu sou o (a) único (a) a defender o que está certo. } \\
\hline \multicolumn{3}{|l|}{ 42. Algumas vezes minha mente fica presa em tantas idéias diferentes que eu não consigo tomar uma decisão ou fazer qualquer coisa. } \\
\hline \multicolumn{3}{|l|}{ 43. Quando eu fico com raiva eu frequentemente "explodo". } \\
\hline \multicolumn{3}{|l|}{ 44. Algumas vezes minha mente fica presa em uma idéia que eu não consigo tomar uma decisão ou fazer qualquer coisa. } \\
\hline \multicolumn{3}{|l|}{ 45. As pessoas não parecem gostar de minhas piadas. } \\
\hline 46. Eu gasto muito tempo pensando nas origens do mundo e da vida. & & \\
\hline 47. Eu sofro de períodos frequentes de muito cansaço e fadiga. & & \\
\hline 48. Eu tenho tido algumas experiências religiosas muito intensas. & & \\
\hline 49. Quase todos os dias eu fico enfurecido (a) por casos em que a justiça não foi feita. & & \\
\hline 50. É inútil contar às pessoas alguma coisa sem dar a elas todos os detalhes. & & \\
\hline 51. Forças poderosas, fora do meu controle, estão atuando na minha vida. & & \\
\hline 52. Minha atividade sexual tem diminuído. & & \\
\hline 53. Eu escrevo ou copio muitas coisas. & & \\
\hline 54. Emoções controlam minha vida. & & \\
\hline 55. Muitas vezes eu sinto como se tivesse feito alguma coisa errada ou prejudicial. & & \\
\hline 56. Eu tenho tendência a quebrar coisas ou machucar pessoas quando fico enfurecido (a). & & \\
\hline 57. Eu, com frequência, me assusto de forma súbita e sem motivo aparente. & & \\
\hline 58. Antes de tomar uma decisão, eu necessito conhecer cada detalhe. & & \\
\hline 59. Algumas vezes eu me sinto tão bem que as idéias vêm à minha mente mais rápido do que posso acompanhar. & & \\
\hline 60. Uma vez que eu começo a conversar com alguém, tenho dificuldade para interromper a conversa. & & \\
\hline 61. Eu não tenho vivido a vida da maneira correta. & & \\
\hline 62. Eu guardo detalhes especiais sobre minha vida e meus pensamentos. & & \\
\hline
\end{tabular}




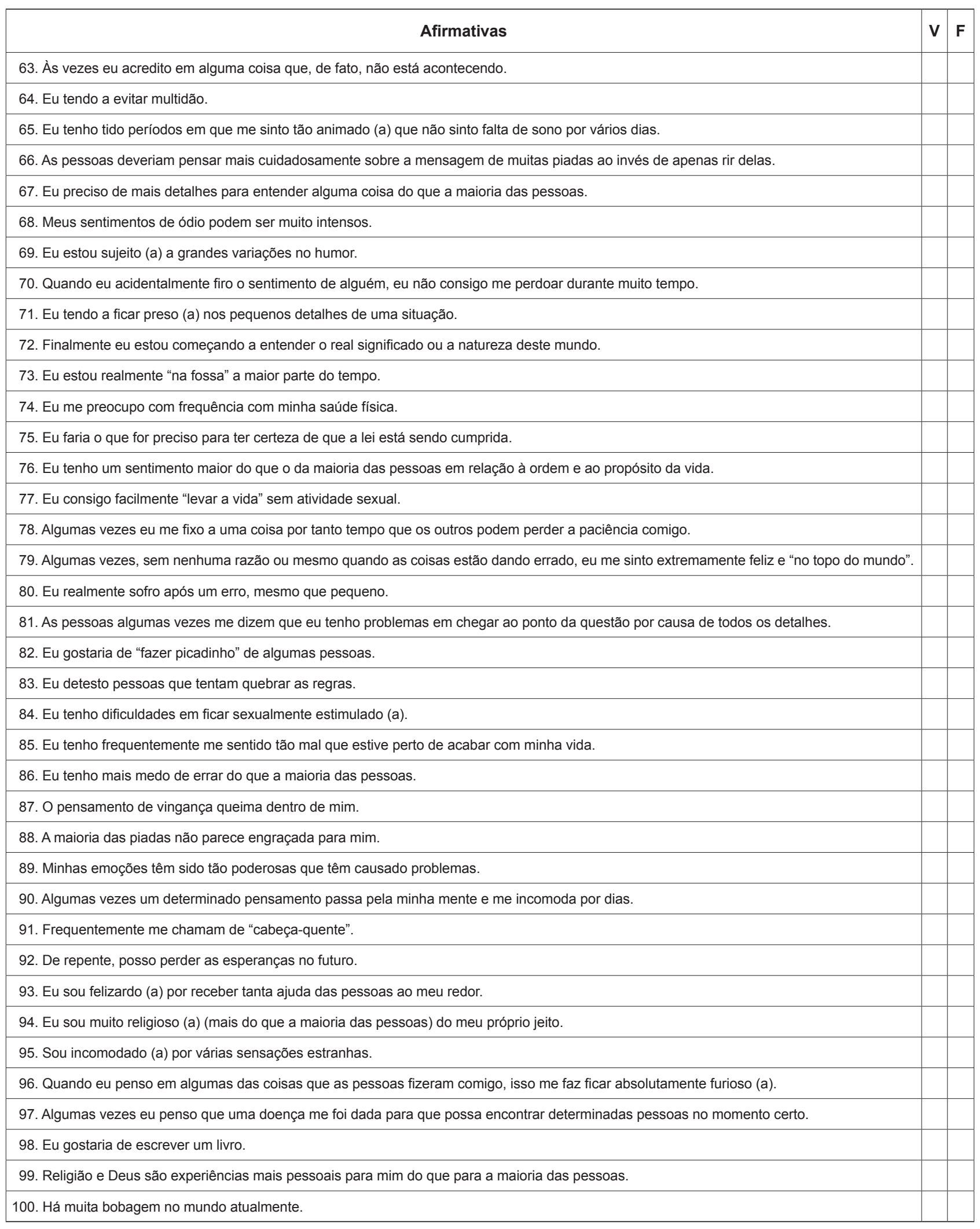

Obrigado por sua honesta e paciente colaboração com o questionário. Por favor, verifique e certifique-se de que todas as perguntas foram respondidas. 